\section{Quade's nonparametric analysis of covariance by matching}

\section{GORDON RAE \\ University of Ulster, Coleraine, Northern Ireland}

A common problem in the behavioral and social sciences is comparing two or more treatments with respect to a response variable $\mathrm{Y}$ when a (possibly multivariate) covariable $X$ is taken into account.

There are two standard approaches to the problem. The first is to adjust for $X$ using classical analysis of covariance. The assumptions required for strict validity of this statistical technique are that under the hypothesis of no treatment effects the conditional distribution of $Y$ given $X$ be (1) normal with (2) expectation linearly dependent on $X$, and (3) variance independent of $X$. Atiqullah (1964) investigated the effects of violations of the first two of these and concluded that the classical analysis of covariance is much less robust than the corresponding analysis of variance, and that the requirement of linearity may be critical. The second standard approach is to hold X constant using procedures based on matching or blocking. Matching, in its simplest version, requires that a subject from one group is paired with a subject from another group for whom the value of $X$ is the same, or at least sufficiently close that a comparison of the subjects' responses can be considered equitable. Although various simple methods are available for dealing with data of this form, matching may be extremely wasteful, particularly if the sample sizes are small or there are several matching criteria, because many of the observations cannot be matched. This disadvantage is avoided by categorizing the covariable and forming blocks, each of which contains all of the observations with covariable values in a given category. Unfortunately, the analysis normally will not be based on the most equitable set of comparisons due to the arbitrariness of categorization (Hoffmann \& Quade, 1983).

In a recent paper, Quade (1982) presented two new approaches to holding $X$ constant: analysis of covariance by matching and analysis of matched differences. These approaches make use of all comparable pairs of subjects and avoid some of the restrictive assumptions of classical analysis of covariance. To apply these procedures, the researcher need only provide a rule for determining whether or not two individuals are comparable. To do this, the researcher specifies a tolerance, the maximum amount by which two individuals may differ with respect to $\mathrm{X}$ and still "match" (or a tolerance for each of its components, if $\mathrm{X}$ is multivariate).

The author's mailing address is: Faculty of Education, University of Ulster, Coleraine, Northern Ireland.
The first procedure, analysis of covariance by matching, is based on the assumption that the marginal distribution of $\mathrm{X}$ is the same regardless of treatments (concomitance assumption) ${ }^{1}$ If , in addition, the conditional distribution of $Y$, given $X$, is the same for each population, then the populations are identical and the samples can be pooled. $A$ predicted $Y$ value, $\hat{Y}$, can then be found for each individual by taking the mean response of other individuals in the combined sample who are matched with that individual on $X$. The differences between the predicted and obtained response values, $Y-\hat{Y}$, are interchangeable and hence, for large sample sizes, it is correct to use them in an ordinary one-way analysis of variance.

The artificial data shown in Table 1 can be used to illustrate the procedure. If the tolerance is $\mathbf{2}$ for matching on the univariate covariable $X$, then the first individual, $a$, is matched with a (by definition), b, c, and e. The mean response for these four observations is $\hat{Y}=(6+5+$ $8+15) / 4=8.5$ and the difference between a's predicted and obtained responses is $\mathrm{Y}-\hat{\mathrm{Y}}=6-8.5=-2.5$. The difference scores for the other subjects are derived in a similar manner. An alternative strategy, which Quade calls "rank analysis of covariance by matching," involves (1) computing for each individual the proportion of matched observations which have lower responses than that individual does minus the proportion with higher responses and (2) performing an analysis of variance on these scores. For example, in Table 1 the first individual, $a$, is matched with a (by definition), b, c, and e (again allowing a tolerance of 2). Of these four matched observations, $b$ has a lower response, $Y$, than a has, but both $\mathrm{c}$ and e have higher responses than $\mathrm{a}$. The corresponding proportions are $1: 4$ and $2: 4$ and the difference $S=-0.25$. The other $S$ values in the Table may be checked similarly.

The second procedure described by Quade, analysis of matched differences, does not require the assumption of concomitance but is restricted to situations in which there are only two groups (which, for convenience, are called the control and treatment groups). This procedure involves finding all of the between-sample matched pairs in the data, including overlapping ones, and counting the number of these pairs in which the treatment member's

Table 1

Small Example to Illustrate Calculations for Analysis of Covariance by Matching

\begin{tabular}{crrrr}
\hline \multicolumn{5}{c}{ Analysis of Covariance by Matching } \\
\hline Subject & $\mathrm{X}$ & \multicolumn{1}{c}{$\mathrm{Y}$} & $\mathrm{Y}-\hat{\mathrm{Y}}$ & $\mathrm{S}$ \\
\hline \multicolumn{5}{c}{ Treatment Group } \\
$\mathrm{a}$ & 10 & 6 & -2.5 & -.25 \\
$\mathrm{~b}$ & 12 & 5 & -4.0 & -.75 \\
$\mathrm{c}$ & 8 & 8 & 1.33 & .67 \\
& \multicolumn{5}{c}{ Control Group } & \\
$\mathrm{d}$ & 14 & 10 & 0.0 & 0.0 \\
$\mathrm{e}$ & 12 & 15 & 6.0 & .75 \\
$\mathrm{f}$ & 7 & 6 & -1.0 & -.50 \\
\hline
\end{tabular}


response is higher than the control member's response, and the number in which it is lower. Subtracting the proportion of pairs in which the treatment member's response is lower from the proportion in which it is higher yields a statistic in the range -1 to +1 , which Quade calls the probability matched difference. The value -1 would indicate that all the treatment members have lower responses, and +1 would indicate that all the treatment members have higher responses. Apart from chance fluctuations in sampling, the two proportions will be equal if there is no treatment effect and the statistic will have the value 0 .

For example, in Table 1, allowing a tolerance of 2 , there are four between-samples matched pairs: $(a, e),(b, d),(b, e)$ and $(c, f)$. In only one of these pairs, $(c, f)$, is the treatment member's response, $Y$, higher than that of the control. In the remaining three pairs the treatment member's response is lower than that of the control. The corresponding proportions are thus $1: 4$ and $3: 4$, yielding a probability-matched difference of $.25-.75=-0.5$.

Another statistic that may be helpful in describing the data is the mean matched difference, which is the mean of the differences between the treatment and the control members' responses over all matched pairs. Quade has shown that both statistics-the probability matched difference and the mean matched difference-are in fact ratios of two U-statistics. Consequently, their standard errors can be readily obtained and used to construct standard normal critical ratios $(Z)$ for significance testing, provided of course that the sample sizes are sufficiently large. If there are more than two groups it is always possible, and may be useful, to calculate matched differences for each pair of groups.

Finally, all of the procedures described above can be readily implemented when $X$ is a discrete variable (for example, political party affiliation or marital status) by allowing a tolerance of zero. If a classical analysis of covariance were to be used, such variables would have to be replaced by dummy variables.

Input. The following items must be input for the program to run: the number of covariates, the number of treatments, the sample sizes (which need not be equal), the tolerances allowed for each covariate, and the values for the covariates and response variable.

Output. The following are given as printed output: summary tables for analysis of covariance by matching and rank analysis of covariance by matching, including sums of squares, mean squares, degrees of freedom and $F$ ratios; and matched differences in mean and probability, with corresponding standard errors and critical ratios.

The sample output shown in the Appendix was based on the cholesterol concentrations (Y) in two groups of women, 11 from Iowa (arbitrarily designated the control group) and 19 from Nebraska (see Quade, 1982, Figure 2).

Allowing a tolerance on the covariable $X$ (age) of 10 years, analysis of covariance by matching and the corresponding rank analysis yielded $\mathrm{F}$ ratios of 1.89 and 1.58 , respectively, which, with $1 / 28 \mathrm{df}$, failed to reach significance at the .05 level. The critical ratios of 1.60 and 1.45 , obtained from the analysis of matched differences in mean and the matched differences in probability, support this conclusion: Tables of the areas under the standard normal distribution indicate that the two-sided $P$ values corresponding to these critical ratios are .11 and .15 , respectively.

Restrictions. The maximum number of covariates and treatment groups are 8 and 10 , respectively. The maximum number of subjects in any group is 100 and the total number of subjects should not exceed 200 . These parameters should be sufficient for most problems but, if necessary, they can be readily altered by modifying the DIMENSION statements.

Computer and Language. The program is written in FORTRAN 77 with list-directed input and was prepared and tested on the University of Ulster VAX-11 computer.

Availability. A source listing may be obtained without charge from the author.

\section{REFERENCES}

Atiqullah, M. (1964). The robustness of the covariance analysis of a one-way classification. Biometrika, 51, 365-372.

Hoffmann, C. C., \& Quade, D. (1983). Regression and discrimination: a case of lack of fit. Sociological Methods and Research, 11, 407-442.

QUADE, D. (1982). Nonparametric analysis of covariance by matching. Biometrics, 38, 597-611.

\section{NOTE}

1. The assumption of concomitance is not required mathematically for the classical analysis of covariance although, as Hoffmann and Quade (1983) point out, it does seem essential for its interpretation.

APPENDIX

\begin{tabular}{|c|c|c|c|c|}
\hline \multicolumn{5}{|c|}{ ANALYSIS OF COVARIANCE BY MATCHING } \\
\hline \multicolumn{5}{|c|}{$\begin{array}{l}\text { GROUP MEANS FOR ADJUSTED SCORES: } \\
\text { GROUP } 1=-12.543 \\
\text { GROUP } 2=r .888\end{array}$} \\
\hline SOURCE & SS & DF & MS & $\mathbf{F}$ \\
\hline $\begin{array}{l}\text { TREATMENTS } \\
\text { WITHIN } \\
\text { TOTAL }\end{array}$ & $\begin{array}{r}2908.14 \\
43061.55 \\
45969.69 \\
\end{array}$ & $\begin{array}{r}1 \\
28 \\
29\end{array}$ & $\begin{array}{l}2908.14 \\
1537.91\end{array}$ & 1.89 \\
\hline \multicolumn{5}{|c|}{ CORRESPONDING RANK ANALYYSIS: } \\
\hline SOURCE & SS & DF & MS & $\mathbf{F}$ \\
\hline $\begin{array}{l}\text { TREATMENTS } \\
\text { WITHIN } \\
\text { TOTAL }\end{array}$ & $\begin{array}{l}0.46 \\
8.23 \\
8.70\end{array}$ & $\begin{array}{r}1 \\
28 \\
29\end{array}$ & $\begin{array}{l}0.46 \\
0.29\end{array}$ & 1.58 \\
\hline
\end{tabular}

MATCHED DIFFERENCES IN MEANS OF GROUPS:

$\begin{array}{llr}\text { GROUP 1 - GROUP 2 } & =28.082 \\ \text { STANDARD ERROR } & =17.499 \\ \text { CRITICAL RATIO } & =1.605\end{array}$

MATCHED DIFFERENCES IN PROBABILITY:

$\begin{array}{ll}\text { GROUP 1 - GROUP 2 } & =0.342 \\ \text { STANDARD ERROR } & =0.236 \\ \text { CRITICAL RATIO } & =1.453\end{array}$

(Revision accepted for publication April 3, 1985.) 